
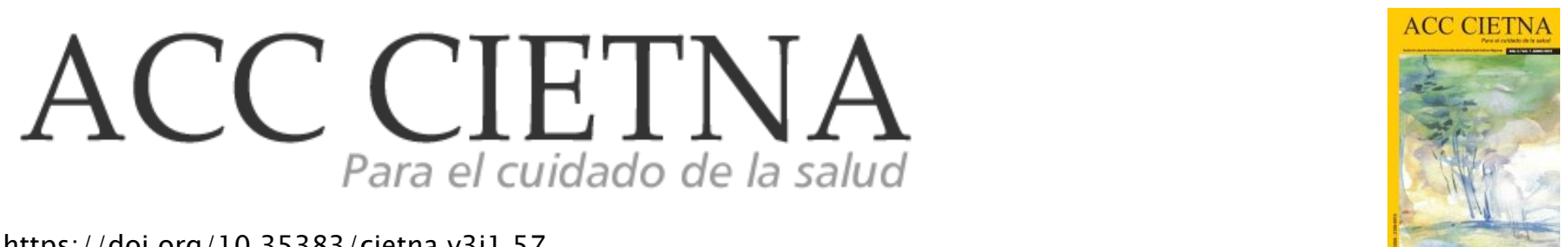

https://doi.org/10.35383/cietna.v3i1.57

\title{
Maltrato laboral: percepciones de las enfermeras en los establecimientos privados de salud, Chiclayo
}

\author{
Jacinto Barrera Julissa Rocio ${ }^{1}$, Reyes Chira Ana María del Rosario², Mesta Delgado Rosio del Pilar ${ }^{3}$
}

INFORMACIÓN DEL ARTÍCULO
Historia del artículo:
Recibido el 14 de enero de 2016
Aceptado el 15 de mayo de 2016

\section{Palabras claves:}

Maltrato laboral

Percepciones

Centros de salud

Enfermera.

\section{RESUMEN}

El maltrato en el sector salud, afecta las relaciones interpersonales y por tanto la organización del trabajo, provocando además, daños físicos o psicológicos, que interfieren con la calidad del cuidado. Es así que se realizó la investigación: "Percepción de las enfermeras sobre el maltrato laboral en los establecimientos privados de salud, con el fin de identificar los tipos de maltrato que reciben dichos profesionales y describir las percepciones de las enfermeras sobre el maltrato laboral. Investigación de tipo cualitativa, con abordaje estudio de caso, cuyos sujetos fueron enfermeras víctimas de maltrato laboral que laboran o hayan laborado en los establecimientos privados de salud. Se tuvo como escenario los establecimientos privados de salud, de la ciudad de Chiclayo; se utilizó como instrumento para la recolección de datos la entrevista semi estructurada, teniendo en cuenta los principios éticos de Sgreccia y de rigor científico de Polit; obteniendo 3 categorías: Percibiendo y tipificando el maltrato laboral en la enfermera; personas generadoras de maltrato laboral a enfermeras en los establecimientos privados de salud y manifestación de sentimientos y reverberaciones del maltrato laboral en la enfermera. Los resultados fueron que el maltrato laboral percibido por las enfermeras es el verbal, psicológico y económico. Además, los principales actores sociales generadores de maltrato laboral son los familiares de los pacientes, los pacientes, enfermeras jefes de los servicios y los directivos de los establecimientos privados de salud.

\footnotetext{
${ }^{1}$ Licenciada en Enfermería. Enfermera del Puesto de Salud La Saucha - Ayabaca - Piura, Perú. Email: julize 810@hotmail.com

2 Licenciada en Enfermería. Enfermera con trabajo independiente, Chiclayo, Perú. Email: areyes@gmail.com

${ }^{3}$ Magíster en Enfermería. Docente en la Escuela de Enfermería de la Universidad Católica Santo Toribio de Mogrovejo, Chiclayo,
}

Perú. Email: rmesta@gmail.com 
Labor abuse: Perceptions of Nurses in Private Health Establishments, Chiclayo

ABSTRACT

Keywords:

Labor abuse

Perceptions

Health centers

Nurse
Abuse in the health sector, affects interpersonal relationships and therefore work organization also causing physical or psychological harm, that interfere with quality of care. Thus, the research was conducted: "Perception of nurses on the labor abuse in private health facilities in order to identify the types of abuse they receive such professionals and describe the perceptions of nurses on the labor abuse. Type qualitative research with case study approach, whose subjects were victims of labor abuse nurses who work or have worked in private health facilities. private health facilities in the city of Chiclayo was played; It was used as a tool for data collection semistructured interview, taking into account the ethical principles of scientific rigor Sgreccia and Polit; obtaining 3 categories: Perceiving and typifying the labor abuse in nursing; persons generating labor abuse nurses in private health facilities and expression of feelings and reverberations of labor abuse in nursing. The results were that the labor nurses perceived abuse is verbal, psychological and economic. In addition, the main social actors generators labor abuse are family members of patients, patients, nurses services chiefs and managers of private health facilities.

\section{Introducción}

El maltrato es un comportamiento deliberado, que provoca, daños físicos o psicológicos entre las personas, y es percibido como un problema de salud pública preocupante ya que afecta a todos los profesionales, adquiriendo múltiples formas y llegando a afectar ámbitos como la familia, amigos y sobre todo el desempeño laboral.1,2

Sabemos que la labor de la enfermera como parte del equipo multidisciplinario es velar por la salud mental, prevenir todo tipo de maltrato, y crear condiciones de autoridad moral favorables para las personas; pero cómo es que ellas podrán brindar estos cuidados y prevenciones si entendemos que las personas no pueden dar aquello que no poseen.

En países de Latinoamérica como: Chile, Argentina,
Brasil, se dieron casos en que el acosador tuvo poder sobre la víctima; la víctima al principio no dio crédito a lo que pasaba, creía que era un malentendido, o pensó que su jefe tuvo un mal día, al prolongarse esa situación, pensó que hizo mal su trabajo y se identificó con la imagen de persona desvalorizada que el acosador le devolvió. ${ }^{2}$

El maltrato laboral en el sector salud, representa la cuarta parte del total de maltrato y en los últimos años ha tomado vital importancia al evidenciarse la manera en la que ha llegado a repercutir en la labor del cuidado de enfermería, originando problemas serios como el abandono del puesto de trabajo, incremento de accidentes en el puesto de trabajo, disminución de la cantidad y calidad del trabajo, lo cual produce costos elevados en la sociedad y en la víctima que ve amenazada su profesión, situación familiar, social, financiera, e incluso su salud. ${ }^{3}$ 
Según apreciación de las investigadoras, los lugares que se volvieron propicios para realizar estos actos de maltrato, fueron aquellas asociaciones conformadas por personas que poseen autonomía económica y administrativa, nos referimos a los establecimientos privados de salud; pues es ahí, donde se perpetúa el acoso a muchos enfermeros, llegando a perjudicar seriamente a los acosados, quienes tienen que aceptar trabajar bajo condiciones que no le permiten pedir un trato igualitario y digno.

Abordar el tema de maltrato laboral, surgió a partir de conversaciones con licenciadas de enfermería, que laboraban en los diferentes establecimientos privados de salud de Chiclayo, las que denotaban a simple vista señales de no sentirse satisfechas laboralmente, pues según sus manifestaciones, eran víctimas de hostigamiento y un bajo reconocimiento económico.

Por otro lado, en un artículo periodístico, ${ }^{4}$ la Decana del Colegio de Enfermeras del Perú, denunciaba al Estado por permitir condiciones laborales y de remuneración, lamentables; explicó que tal situación se vive en las dependencias de clínicas privadas, donde pese a que la situación es más auspiciosa para brindar mejor trato a las enfermeras, esto no sucede.

Podemos decir que el maltrato laboral deja huellas indelebles, que pueden ir desde estrés postraumático, hasta una vivencia de vergüenza recurrente o incluso cambios duraderos de su personalidad. La desvalorización persiste aunque la persona se aleje de su acosador, la víctima lleva una cicatriz psicológica que la hace frágil, a vivir con miedo y a dudar de lo que realiza.

Nos resultó inquietante observar que en los establecimientos privados de salud de la ciudad de Chiclayo el personal de enfermería acepte pasivamente, ya sea por necesidad o por falta de trabajo, el maltrato de todo tipo considerándolos como "parte del trabajo", "rutina diaria" o como un "juego" de profesionales de salud de mayor jerarquía, sin tomar en cuenta que esto puede tener un efecto nocivo en la salud personal y en el desempeño laboral.

Por otro lado, según informe del año 1998 de la Organización Internacional del Trabajo (OIT), citado por la OMS, 1 se llegó a la conclusión de que el maltrato en el lugar de trabajo también tiene carácter mundial, el cual atraviesa las fronteras, los contextos de trabajos, los grupos profesionales, siendo la profesión de enfermería de alto riesgo ocupacional en el lugar de trabajo, este se manifiesta o se percibe de diversas maneras; el maltrato verbal que produce intimidación y daños psicológicos, el abuso que puede llegar a la agresión física, y en algunas situaciones al acoso sexual.

El Consejo Internacional de Enfermería (CIE), 2 en 1999 demostró que entre el personal de salud, las enfermeras son las más expuestas al riesgo de maltrato laboral, en el 2001 , con una incidencia de $87 \%$ de casos en las diversas unidades hospitalarias.

Además, estudios publicados también por el CIE en ese mismo año con respecto al recurso humano de enfermería, demostró cómo han sido violentadas y agredidas las enfermeras en su ambiente laboral.

Las realidades mencionadas por la OIT y el CIE, no son ajenas a lo que han vivido hoy en día las enfermeras en los establecimientos privados de salud las cuales fueron víctimas de maltrato laboral de múltiples formas, no solo por parte de pacientes y enfermeras jefes; sino también, por directivos, es por ello que se planteó la pregunta de investigación científica:

¿Cuál es la percepción de las enfermeras sobre maltrato laboral en los establecimientos privados de salud - Chiclayo 2011 ?

Para lo cual se plantearon los objetivos siguientes: 
Identificar los tipos de maltrato laboral en los profesionales de Enfermería de los establecimientos privados salud y Describir las percepciones de las enfermeras sobre el maltrato laboral.

\section{Metodología}

Las investigadoras realizaron el trabajo de investigación de tipo cualitativo, la cual consiste en la recolección y análisis sistemático de materiales narrativos, tal como sucedió con los discursos de las enfermeras víctimas de maltrato laboral, que encerró un alto contenido subjetivo, entre sus principales características pueden mencionarse: Enfocaron el estudio del objeto en su totalidad sin recurrir al análisis de variables aisladas. Por otro lado, en un sentido amplio, la investigación cualitativa produjo datos descriptivos: las propias palabras de las personas, habladas o escritas, y la conducta observable. ${ }^{5}$

Los estudios cualitativos están preocupados por el entorno de los acontecimientos, y centran su indagación en aquellos contextos naturales, o tomados tal y como se encuentran, más que reconstruidos o modificados por el investigador, en los que los seres humanos se implican e interesan, evalúan y experimentan directamente. 6

Tuvo como abordaje metodológico el estudio de caso, descrito por Pérez Serrano como un camino para la construcción de conocimiento basado en la idea de que al analizarse con detalle cada unidad de un grupo determinado, se pueden comprender sus características en profundidad. ${ }^{7}$

La presente investigación tuvo como sujetos de estudio, a 12 enfermeras víctimas de maltrato laboral que laboran o hayan laborado en establecimientos privados de salud de la ciudad de Chiclayo.

Se desarrolló en los diferentes establecimientos privados de salud, entre ellos el Hospital de Clínicas, Hospital Metropolitano; los cuales se encuentran ubicados en la provincia de Chiclayo del departamento de Lambayeque, en su común denominador están conformadas por profesionales de la salud médicos quienes forman grupos de accionistas bajo la reglamentación de estatutos que rigen un consejo directivo, una junta de accionistas y siendo uno de sus fines primordiales brindar servicios de salud con atención de calidad. Su ubicación se encuentra en avenidas centrales y de fácil acceso a la población chiclayana, cuentan con una infraestructura que oscila entre 5 a 15 años de antigüedad.

Para la aprobación de la tesis por la escuela de enfermería de Universidad Católica Santo Toribio de Mogrovejo Chiclayo - Perú y validado el instrumento de recolección de datos mediante la ejecución de la prueba se procedió a recoger los datos de las enfermeras de los diferentes establecimientos privados de salud de Chiclayo del departamento de Lambayeque, así como el respectivo permiso por cada institución. Las entrevistas se ejecutaron de manera alternada, de acuerdo a su disponibilidad; éstas se desarrollaron en ambientes preestablecidos a su solicitud, acondicionados para mantener la privacidad misma siendo la mayoría de las entrevistas realizadas en las casas de las enfermeras, estos ambientes fueron con una adecuada ventilación e iluminación, las enfermeras fueron amables, colaboradoras, accediendo a ser entrevistadas; con cada una de ellas fue una experiencia diferente, estando las investigadoras dispuestas a acceder a que nos proporcionen una entrevista en el lugar que escogieran, siempre tratando de no incomodar.

La actividad prioritaria de la entrevista fue explicar los objetivos de la investigación para su autorización, se asintió preguntas para aclaraciones pertinentes, observándose que en la mayoría había una resistencia a firmar el consentimiento informado que consigna su nombre y firma; se aclaró que sería seriamente guardado por las investigadoras y sólo se manejaría su seudónimo para lo cual se utilizó 
seudónimos de flores siguiendo con los principios de ética de Elio Sgreccia.

La entrevista que se aplicó a las enfermeras víctimas de maltrato laboral fue en un tiempo de 3 meses debido a las múltiples actividades que ellas realizaban y lo imprevisto de la disponibilidad de su tiempo.

Concluida la recolección de datos, se procedió a la transcripción fidedigna de la entrevista aplicada a las enfermeras, las mismas que se realizaron de manera inmediata a la conclusión de la misma, cuya transcripción por discurso llevó un promedio de 3 horas, para poderlos transcribir tal cual fueron relatados por los sujetos de investigación; luego se llevó a cabo la lectura y relectura de los discursos transcritos, para identificar unidades de análisis significativos que nos permitan más adelante poder identificar, describir y analizar las experiencias sobre maltrato laboral en lo expuesto por las enfermeras sujetos de estudio.

Realizar el análisis implicó el reposo y asimilación de los discursos, de manera pormenorizada, y considerándose para ello las 3 etapas propuestas por Burns. ${ }^{8}$

La primera es el pre análisis, siendo la etapa de organización del material a analizar, en la que se diseñan y definen los ejes del plan que permite examinar los datos y realizar efectivamente el análisis.

En el estudio las investigadoras realizaron la revisión de las entrevistas escuchándolas, hasta que se vio inmersa en los datos, para ello se repitieron con la frecuencia necesaria para poder no solo entender sino también internalizar los discursos, de tal manera que la familiaridad con los mismos permitieron una mejor contextualización semántica, que facilitó su análisis a la luz del conocimiento de las bases teóricas.

La segunda etapa es la codificación, la cual consiste en realizar una transferencia de los datos brutos a los datos útiles. Burns ${ }^{8}$ refiere que en esta etapa se realizará el filtro de los discursos, fragmentación de los mismos y la extracción de unidades significativas, estas, en el presente trabajo, se obtuvieron de los discursos previamente llevados del Emic al Etic, utilizando el método de recorte e identificación por números. Al fragmentar los discursos se obtuvieron 96 fragmentaciones significativas, relacionadas directamente con los sujetos y objetos de estudio; seguidamente, se procedió a agrupar por similitud semántica, dando lugar a la codificación y agrupamiento que tuvo un constructo de hasta cuatro intentos, quedando una propuesta final, después de haber intentado "encarcelar" los fragmentos con la mayor propiedad posible de similitud.

Finalmente la última etapa fue la de categorización, consiste en la organización y clasificación de las unidades obtenidas en grandes categorías y subcategorías.

Las investigadoras una vez obtenidos los datos, procedieron al análisis temático relacionado al contenido, codificando y clasificando los temas que dieron origen a las grandes categorías y subcategorías preliminares. Finalmente al término de la revisión de los discursos y de la literatura emergieron las siguientes categorías: Percibiendo y tipificando el maltrato laboral en la enfermera. Personas generadoras de maltrato laboral a enfermeras en los establecimientos privados de salud con subcategorías subtituladas: Pacientes principales generadores de Humillación hacia las enfermeras, Vilipendio laboral originado por familiares y Maltrato laboral producido por el equipo de salud y directivos. Y como última categoría Manifestación de sentimientos y reverberaciones de maltrato laboral en la enfermera.

En la investigación se consideraron los siguientes criterios éticos: El primer principio considerado es el valor fundamental de la vida humana; que está estrechamente unido a la concepción ontológica de la corporeidad, apoyada y desarrollada en todos 
los demás valores de la persona. La inviolabilidad de este principio fue un signo y una exigencia de la inviolabilidad misma de la persona, por tanto la persona fue un sujeto de moral y un individuo respetado. 9 Este primer principio fue de gran utilidad para las investigadoras pues la persona humana es considerada siempre como sujeto y no como objeto, donde los sujetos de investigación que participaron durante la investigación fueron considerados como personas en su totalidad, por lo cual las investigadoras se abstuvieron de utilizarlos como medio para el logro de sus objetivos, para esto con la información obtenida, se fue muy cauto, su identidad se mantuvo en anonimato para lo cual se utilizaron seudónimos de flores y se guardó la debida confidencialidad lo cual implicó que los participantes del estudio fueran respetados en su integridad y dignidad humana.

Otros de los principios considerados en la presente Investigación es el de la libertad y la responsabilidad; porque respetar la libertad significa respetar la vida, ello concierne conocer y poder elegir responsablemente hacia sí mismo y hacia los demás. Es por tal motivo que se informó a los participantes sobre lainvestigación a realizar, se respetó en todo momento la decisión de participar en forma voluntaria y libre en la investigación. Por tal motivo el contacto con las personas que participaron en la investigación, se realizó de manera personal y explicando el estudio y vinculación, siempre con el compromiso y responsabilidad de proteger la identidad a los sujetos de estudio y hacer uso de los relatos solo para fines de investigación.

Principio de Sociabilidad - Subsidiariedad, según el cual la persona está inserta en una sociedad que debe tender al bien común: cada hombre es responsable de su salud y de la salud de los demás, complementariamente. La subsidiaridad implica respetar a la persona atendiendo sus necesidades sin influenciar en su capacidad de decisión. Se procuró extremar los posibles beneficios y minimizar los posibles riesgos hacia los sujetos de investigación. Así mismo se respetó la autonomía de cada uno de ellos atendiendo a sus necesidades sin sustituirles de decidir y de actuar.

Los criterios de rigor científico que se utilizaron en el presente estudio cualitativo fueron la credibilidad, la confirmabilidad, la transferibilidad. 10

La credibilidad se logra cuando el investigador, a través de observaciones y conversaciones prolongadas con los participantes en el estudio, recolecta información que produce hallazgos que son reconocidos por los informantes como una verdadera aproximación sobre lo que ellos piensan y sienten.

Así entonces la credibilidad se refiere a como los resultados de una investigación son verdaderos para las personas que fueron estudiadas y para otras personas que han experimentado o estado en contacto con el fenómeno investigado.

Otro de los principios que se tuvo en cuenta la confirmabilidad, se refiere a este criterio como la habilidad de otro investigador de seguir la pista de lo que el investigador original ha hecho. Para ello se realizó un registro y documentación completa de las decisiones e ideas que las investigadoras obtuvieron con el estudio. Esta estrategia permite que otro investigador examine los datos y pueda llegar a conclusiones iguales o similares a las del investigador original siempre y cuando tengan perspectivas similares.

Y por último tenemos al criterio de transferibilidad, este criterio se refiere a la posibilidad de extender los resultados del estudio a otras poblaciones. Indican que se trata de examinar qué tanto se ajustan los resultados con otro contexto.

\section{Resultados, análisis y discusión}

I: Percibiendo y Tipificando el maltrato laboral en la enfermera 
Para las enfermeras percibiendo es distinguido como el proceso de recibir mediante los sentidos las imágenes, impresiones o sensaciones externas, - aquel proceso mediante el cual se puede comprender y conocer algo. Así se llega hacia una idea más clara, ésta puede destinarse a hacer mención a un determinado conocimiento, a una idea o a la sensación interior que surge a raíz de una impresión material derivada de nuestros sentidos.

La primera categoría pone énfasis en las percepciones y tipifica los tipos de maltrato laboral en el que la enfermera de los establecimientos privados de salud diariamente está expuesta, a hechos en que el maltrato puede estar dirigido hacia ellas y puede ser directa o implícita.

Dentro del campo de la salud la enfermería constituye un importante espacio en la sociedad sobre todo porque se trata del esfuerzo que los profesionales de esta área sin importar los horarios en que laboran, turnos diurnos y nocturnos, desempeñan su labor, pues la mayoría desconoce los momentos de riesgo de este grupo humano, que mantienen a diario una dedicación por el trabajo, estos riesgos están presentes en cada ocasión generada en el desarrollo de sus funciones como profesionales de salud.

Sabemos que el trabajo es un espacio esencial para el despliegue de la identidad, la creatividad y la autorrealización de los individuos, sin embargo deja de funcionar de esta manera para transformarse en un generador de maltrato en determinados contextos, anulando los lazos de cooperación entre los trabajadores y dirigiéndolos a dinámicas de maltrato hacia los demás transformándose en un espacio desmotivador tanto de los pacientes como de los propios trabajadores $^{11}$, es así que los tipos de maltrato que perciben las enfermeras en el sector privado son: El maltrato verbal y psicológico que se ha convertido en una realidad demasiado frecuente en el sector salud, este tipo de maltrato percibido por el profesional de enfermería va desde gritos por parte de personas que exigen ser atendidas en el instante por la enfermera, la cual sin darse cuenta empieza a admitirlo como algo rutinario, que se da a diario, son víctimas de actitudes violentas incluso por el personal del equipo de salud dentro de los servicios donde brindan cuidado. Ya que muchos creen que el único trabajo de las enfermeras en los establecimientos privados de salud es sencillamente estar pendiente del llamado del paciente y de los familiares. 12

"Entré a realizar mis cuidados al paciente, llegó el médico y me comenzó a gritar idonde has estado, te estoy buscando, apúrate con lo que estás haciendo y me vienes a ver porque voy a pasar visita a mis pacientes"...Margarita

Las enfermeras que laboran en los establecimientos privados de salud además han tipificado otro tipo de maltrato y este es el maltrato económico. Entendido como la actuación, acto, o represión, ejercido con todo lo referente a la percepción u obtención del dinero en un individuo, este tipo de maltrato es ejercido por personas, instituciones privadas, e incluso sistemas sociales a través de la remuneración desigual, y el no pago de las prestaciones.

Al analizar la constitución política encontramos en el artículo 24 derechos del trabajador lo siguiente: el trabajador tiene derecho a una remuneración equitativa y suficiente, que procure, para él y su familia el bienestar material y espiritual; además el pago de la remuneración y los beneficios sociales del trabajador tiene prioridad sobre cualquier otra obligación del empleador; y las remuneraciones mínimas se regulan por el estado con participación de las organizaciones representativas de los trabajadores y de los empleadores. ${ }^{13}$ Los recursos humanos son un elemento clave y la principal ventaja competitiva que poseen los servicios de salud, es por ello que es preciso brindar las condiciones necesarias para lograr un óptimo desenvolvimiento $\mathrm{y}$, por ende, el logro de los objetivos en materia de salud propuestos a distinto nivel, es allí donde las remuneraciones, los 
términos contractuales y otros tipos de incentivos cumplen una función importante.

En nuestro país existen diversas modalidades de contratación de personal, así como instituciones que brindan plazas de trabajo para los diferentes profesionales de la salud, siendo el Ministerio de Salud (MINSA) y la Seguridad Social (EsSalud) las que reúnen el mayor número de estos. Si bien ambas son instituciones estatales, en el primer caso se encuentra bajo un régimen público y en el segundo bajo uno de tipo privado. Los regímenes de relación contractual son diversos, estableciéndose dos grandes grupos de trabajadores: el personal nombrado y el personal contratado; en este último grupo existen diversas modalidades de contratación regidas por legislación propia de cada sector, que generan a su vez diversas escalas remunerativas con composiciones variables que incluyen beneficios e incentivos relacionados con la productividad $y$ otras formas, como mecanismo para contrarrestar el hecho de que no han existido incrementos salariales satisfactorios en los últimos años, estando ausentes en la Ley del Presupuesto Nacional. Las remuneraciones de los profesionales de enfermería en el MINSA fluctúan entre los $\mathrm{S} /$. 900 (CLAS) y de $S / 1000$ a S/1500 en CAS y en EsSalud oscilan entre S/. 2300 y S/. 2500 de salario mensual, con el salario básico, bonos y guardias. 14

Para las investigadoras otra viene siendo la situación en el caso de los establecimientos privados de salud, ya que el trabajo de las enfermeras y el ambiente donde lo desarrollan pareciera no ser el más propicio para derivar en satisfacción laboral; según lo manifestado por las enfermeras de los diferentes establecimientos de salud privados de la ciudad de Chiclayo la modalidad por la que ingresan a trabajar es muy diferente a los antes mencionados (MINSA y ESSALUD) ya que al inicio deben realizar una llamada pasantía no remunerada de 10 a 15 días e incluso llega a 1 mes aproximadamente, la cual terminada conlleva a un puesto de retén y a la cobranza de sus salarios mediante recibos por honorarios en un tiempo aproximado de 2 a 3 años bajo amenaza de ser relevadas de sus puestos en cualquier momento, y después de todo este proceso recién ser reconocidas en la planilla.

Al mismo tiempo, existen las bajas remuneraciones que a veces las obliga a desempeñar más de un trabajo teniendo que asumir largas jornadas con el consiguiente desgaste que ello significa agravar su situación; las remuneraciones percibidas por las enfermeras de los establecimientos privados solo alcanzan el sueldo mínimo vital de 750 soles mensuales; los pagos por guardias nocturnas circundan entre 60 soles por turno, no se les reconoce los días domingos y solo tienen derechos a las vacaciones pagadas las enfermeras que se encuentren en planilla. Tal como se aprecia en los siguientes discursos

"Con el sueldo que nos pagan de 750 soles no nos alcanza, porque muchas de nosotras tenemos familia y responsabilidades en casa, o en mi caso que quiero seguir cursos o una maestría pero por las bajas remuneraciones trabajo en dos establecimientos privados para seguir creciendo profesionalmente"....Clavel

II: Personas generadoras de maltrato laboral a enfermeras en los establecimientos privados de salud

En los establecimientos privados de salud se conoció por medio de nuestros sujetos de estudio que las enfermeras reciben maltrato laboral por diferentes personas que se encuentran dentro del contexto tales como los pacientes que acuden a una atención, más que todo a un servicio y que en cierto punto generan exigencia contractual dado que ellos hacen un reembolso económico.

Así mismo los familiares que acompañan a los pacientes demandan una atención de inmediato llegando muchas veces al abuso y al insulto, así como miembros del equipo de salud que de una $u$ 
otra manera se encargan de generar malestar en la enfermera.

El maltrato laboral por parte de estas personas, se produce de forma sistemática y recurrente durante un tiempo prolongado pero no es considerada de gran importancia por parte de la enfermera ya que en muchos casos vemos que los asume como parte de la labor profesional, surgiendo la sub categoría:

\subsection{Pacientes principales generadores de humillación hacia las enfermeras}

El sector salud como ámbito laboral no escapa a la presencia de maltrato, siendo motivo de preocupación en especial para enfermería; se considera que los principales factores que contribuyen al maltrato contra las enfermeras en su campo laboral son: tener que desempeñar su trabajo de manera aislada, el contar con escaso personal técnico, tener que tratar con personas que pueden estar estresadas o afligidas.

La agresión de las enfermeras es perpetrada casi exclusivamente por los pacientes. Las enfermeras responden de distintas maneras cuando ocurre un episodio de maltrato, y esta diferencia en su reacción depende de: tipo de personalidad, y reacciones inmediatas.

El maltrato puede variar de sumamente pasivo a sumamente activo, a través de un proceso continuo que va desde aceptar, evitar, defenderse verbalmente, negociar, hasta defenderse físicamente. ${ }^{15}$

“El Paciente gritándome.... Señorita atiéndame rápido para eso yo pago mi plata, por mi come, esta acá trabajando en esta institución así que tiene que atenderme bien y rápido"...... Azucena

La agresividad de pacientes hospitalizados casi siempre denota ansiedad, por ello un gran porcentaje de denuncias formuladas contra las enfermeras se evitarían si la relación enfermerapaciente se hiciera con un correcto manejo de la comunicación entre ambos. A pesar de ello, es muy difícil evitar la aparición de situaciones de maltrato, hay que aprender a prevenir la agresión, saber percibirla incluso cuando aún sólo se manifiesta de manera encubierta tal como se evidencia en los siguientes discursos:

\subsection{Vilipendio laboral originado por familiares}

La sociedad actual en la que se desenvuelve la enfermera, ha cambiado durante las últimas décadas, ahora con la sociedad consumista, el estrés y otros factores como el maltrato se ha transformado poniendo en riesgo la estabilidad laboral, emocional y hasta la salud del personal profesional de enfermería, traduciéndose en bajo rendimiento laboral en la que la enfermera se ve "sometida" ante las demandas de los familiares. ${ }^{16}$

Es por ello que decimos que los profesionales de enfermería de los establecimientos privados de salud no escapan a la intolerancia general y son atacados física o verbalmente por algunos familiares como lo demuestra el siguiente discurso:

“...Al star de enfermería se acercó el familiar de un patient y golpeando la mesa me dijo: ¿oiga que está haciendo? trabaje, le toca la medicina a mi familiar; dele su pastilla rápido, usted está perdiendo el tiempo escribiendo me voy a quejar que no cumple con sus funciones..."Gardenia

Para los familiares de los pacientes la falta de atención inmediata a necesidades entre ellas las de aseo y/o confort, es uno de los factores que ocasiona que se comporten de una manera ofuscada con el profesional de enfermería de los establecimientos privados, ya que según ellos es un tiempo prolongado que demora el personal profesional de enfermería en atender al llamado de su solicitud, calificando esta situación como una "mala atención" pues se justifican en que al realizar un desembolso económico a la entidad de salud merecen tener la mejor de las atenciones de 
manera inmediata y sin reclamo alguno por parte del personal de enfermería.

Sin embargo no imaginan las múltiples funciones que deben realizar las enfermeras, ya que en su mayoría de veces los servicios privados de salud son saturados y esto se relaciona con la escasez de personal para la atención de los pacientes hospitalizados; ya que el recurso humano disponible en los establecimientos privados es limitado, y escapa de sus manos atender a tiempo a todos los llamados de los pacientes que requieren atención.

Sabemos que el profesional de enfermería debe priorizar los cuidados a pacientes con mayores problemas de salud, sin embargo esto no es entendido por los familiares de los pacientes quienes optan por agredirlas verbal, psicológica y hasta físicamente por el simple hecho de no esperar para el retiro de la chata $u$ otras actividades de menor importancia.

Por ello resulta frustrante para el profesional de enfermería tener que lidiar día a día con los familiares de los pacientes quienes molestos se expresan hacia ellas con el mínimo respeto debido, tomando muchas veces actitudes ofensivas amenazando con demandarlas o presentar quejas a sus superiores (jefa de enfermeras) si no se hace lo que ellos desean; frente a ello las enfermeras solo atinan a bajar la cabeza y continuar con sus labores pues temen perder su puesto de trabajo.

2.3 Maltrato laboral producido por el equipo de salud y directivos.

Tiene lugar en el seno de una organización, por lo que uno de los factores implicados en su desarrollo y mantenimiento es el contexto de la organización, pues en función de cómo sea ésta, puede incrementar o disminuir la posibilidad de que aparezca maltrato laboral.

El proceso de maltrato laboral se ve favorecido por una pobre organización del trabajo, por una deficiente gestión de los conflictos y por un clima laboral propicio al maltrato, así mismo el maltrato también es producido por otros personajes que integran el equipo de salud, como el médico, la enfermera jefe del establecimiento privado, el personal directivo, y administradores de las nstituciones. ${ }^{17}$

“...Me acerqué a la oficina del administrador y le dije: quiero saber porque me están sobrecargando de trabajo, deme una explicación.... en ese momento el administrador se paró (mirándome y frunciendo el ceño) cerró la puerta y no quiso atenderme, regresé a mi servicio indignada y en el transcurso del día me hicieron llegar un documento para que al día siguiente fuera a reportarme a la otra sede de la institución"... Clavel

La cruel realidad es que detrás se escondan hechos que inquietan por la exposición a un mundo social tan deteriorado por el maltrato $y$ por las experiencias de enfermedad que afrontan. La falta del personal puede aumentar el riesgo de maltrato debido a que suele alargar los períodos de espera para brindar la atención y que las enfermeras estén solas con los pacientes. Así mismo un bajo apoyo por parte de la jefa de enfermería, la sobrecarga de trabajo, y unas pobres relaciones con otros profesionales son factores que pueden aumentar el estrés en el lugar de trabajo y también el riesgo de agresión. 18

Se entiende por establecimiento privado a una entidad destinada a prestar servicios de salud, a personas que presenten alteración de su hemodinamia las 24 horas del día para lo cual debe de contar con personal calificado, equipos e infraestructura adecuada, la cual puede ser bajo la modalidad de internamiento o ambulatorios, está compuesta por un equipo multidisciplinario de profesionales de salud los cuales debería procurar entre ellos un ambiente cálido y de confianza para poder desarrollar sus labores complementándose unos a otros, sin embargo eso está quedando fuera de nuestra realidad ya que el egocentrismo por parte de los demás profesionales de salud está 
generando un sentimiento de superioridad en ellos, ocasionando un malestar en la enfermera ya que deja de ser parte de un complemento importante en el equipo de salud para pasar a ser un instrumento al cual se le debe tratar sin ninguna consideración especial ${ }^{18}$ como se presenta en los siguientes discursos:

“...La enfermera jefe de la clínica me cambiaba el rol, aumentándome la sobrecarga laboral, buscaba la forma de aburrirme, hasta que dije ya no quiero trabajar más aquí y renuncié"...Clavel

Si se realizara una buena toma de decisiones, la que debe ser compartida y debe buscar el beneficio de todos los miembros de una empresa, sin embargo algunas decisiones en la práctica cotidiana están frecuentemente expuestas a escenarios que pueden generar gran incertidumbre y a partir de los cuales se pueden tomar decisiones que generan un impacto negativo, en este caso dichas decisiones son tomadas por parte de los directivos de los establecimientos privados quienes se han vuelto también generadores de maltrato laboral, ya que en su mayoría utilizan la política de puertas cerradas, tomando decisiones apresuradas e imponiéndolas a las enfermeras.

III: Manifestación de sentimientos y reverberaciones de maltrato laboral en las enfermeras

Los sentimientos son las reacciones a acontecimientos y hechos de la vida, son un claro indicador de la personalidad del sujeto, que expresan el significado íntimo de lo que está ocurriendo en nuestra vida social.

El Maltrato laboral es un gran riesgo para la salud mental y física de las enfermeras y puede causar deterioro de los cuidados dispensados a los pacientes. Sólo cuando los empleadores, el personal profesional de enfermería y los pacientes tomen en serio los incidentes de maltrato laboral estos riesgos se reducirán por completo de los lugares de trabajo del sector salud. Los efectos del maltrato son inquietantes y comprenden una amplia gama de consecuencias derivadas de dichos actos, las que incluyen desde lesión física, depresión, temor, estrés, pérdida autoestima, siendo también de gran importancia el que puedan comprometer la calidad de los cuidados prestados. ${ }^{19}$

El cansancio, las escasas posibilidades de capacitación debido al sistema de turnos y la escasez de personal traen como consecuencia fatiga y desmoralización entre estos profesionales, el deber de realizar muchas tareas cuya complejidad es inferior a su formación y que comprometen su tiempo, le impiden concretar su función fundamental, que es junto al paciente en la entrega de atención y cuidados de éste.

Por otro lado, los turnos le dificultan la vida matrimonial y familiar, ya que éstos no coinciden con las rutinas normales de trabajo, por lo que la enfermera requiere de muchos esfuerzos para compatibilizar sus actividades con las de su familia.

Además, las bajas remuneraciones que a veces la obliga a desempeñar más de un trabajo teniendo que asumir largas jornadas con el consiguiente desgaste que ello significa agravar la situación.

Nos resulta inquietante observar en algunos casos, como el personal de enfermería acepta pasivamente maltratos de todo tipo considerándolos como parte del trabajo, o la rutina puesto que no se atreve a defenderse y no llega a comunicar esta situación, sin tomar en cuenta que todo ello afecta de manera nociva tanto a su salud como a su desempeño laboral, como se da a conocer en el siguientes discurso:

"...Me sentí triste, al mismo tiempo con rabia de querer responderle al médico, porque llegué con todas las ganas de trabajar, la voluntad de hacer las cosas bien, pero gritándome desde que inicié a trabajar en mi turno no fue bueno para mí...Gardenia 
Las enfermeras que son víctimas de maltrato experimentan diversos sentimientos que afectan su estado emocional generando en su ser la impotencia, e inconsolable dolor emocional que resulta de no poder remediar una situación o circunstancia desagradable. ${ }^{19}$ Las enfermeras ante las diferentes situaciones que se le han presentado en su labor cotidiana de cuidar manifiestan sentir ese sentimiento de incapacidad para enfrentar dichas situaciones llegando a pasarlas por alto hasta quedar en el olvido.

“...Sentí impotencia, ira de que el médico me grite y no poder responderle, en ese momento sentí molestia conmigo misma porque me hubiera podido defender sentí mucha cólera, porque la verdad lo hizo sin razón"... Margarita

Los efectos ocasionados por el maltrato laboral hacia la enfermera han causado múltiples secuelas las cuales se ven reflejadas no solo en el momento de brindar un cuidado de calidad a los pacientes, sino que ha penetrado en el fondo de ella sintiéndose poco capaz no solo en su trabajo sino también en su hogar. Creando en ellas una cierta incomodidad y desmotivación, que se originan por diversas razones que tienen que ver con la empresa, el entorno laboral, y el trabajador en sí, sea cual sea la causa, es necesario asumir la desmotivación como algo propio, independientemente de lo que suceda alrededor; pero en la labor de la enfermera resulta difícil poder sobrellevar estas situaciones ya que al recibir agresiones por parte de las personas que cuidamos o de sus familiares generan una total desmotivación irremediable, como en el caso de los pacientes, siendo nuestros sujetos de cuidado resulten ser nuestros principales desmotivadores. ${ }^{20}$

La ira se ha considerado como una emoción des adaptativa e incluso destructiva, personal y socialmente. El enfoque actual la considera resultado de un proceso evolutivo que prepara al organismo para respuestas de ataque ante daños inminentes, como tal tiene una función de supervivencia del individuo, suele ir acompañada de manifestaciones faciales que actúan como un sistema de aviso y de una actitud del sistema nervioso autónomo y activación corporal que amenaza con irrumpir en la seguridad física de terceras personas y del contexto social. 20

\section{Conclusiones}

Las enfermeras en los establecimientos privados perciben el maltrato laboral como aquel acto $u$ acción negativa que genera un impacto perjudicial en su labor de cuidar, pues perturba su vida de manera significativa. Así mismo lo tipifican en Maltrato verbal el cual va desde gritos, insultos, amenazas; Maltrato psicológico que afecta no solo al autoestima de la persona, también a su estabilidad emocional; y Maltrato económico pues tienen un bajo reconocimiento económico en sus haberes.

En los establecimientos privados el maltrato laboral se genera principalmente por los pacientes, que acuden a ser atendidos y exigen atención inmediata del personal de enfermería dado que hacen un reembolso económico, seguido de los familiares que acompañan a los pacientes que muchas veces llegan al maltrato emocional y físico, así como insultos, burlas hacia las enfermeras y por último por parte del equipo de salud y directivos de los establecimientos quienes actúan y deciden sin tomar en cuenta opiniones del personal de enfermería y solo actuando a su favor.

Los pacientes son los principales generadores de humillaciones a las enfermeras los cuales actúan con gritos y exigen de manera agresiva que atiendan a su llamado sin importarles las múltiples actividades que realizan las enfermeras.

Las enfermeras reciben maltrato laboral por parte de familiares de los pacientes los cuales parten desde actitudes, palabras que atentan contra su personalidad, dignidad y llegan a una degradación moral que daña el estado de ánimo, su propio valor y la capacidad de tomar decisiones. 
El maltrato laboral producido por el equipo de salud y directivos como el médico, la enfermera jefe de clínica, viene siendo considerada erróneamente por los profesionales de Enfermería como una cuestión de adaptación y aceptación, y según ellos forma parte de la rutina, dejando así de actuar como parte de un equipo multidisciplinario de profesionales de salud y de complementarse unos a otros, ocasionando un malestar a la enfermera ya que deja de ser parte de un complemento importante para pasar a ser un instrumento al cual se le trata sin ninguna consideración, abriendo un espacio de distanciamiento y resentimiento, que se percibe como actitud de rechazo debido a la sensación de insatisfacción y frustración en el ámbito laboral; esto a su vez de la mano con comentarios despectivos que se perciben como las críticas.

Las enfermeras manifiestan sus sentimientos al ser maltratadas con tristeza, cólera y repercuten deteriorando los cuidados dispensados a los pacientes así como efectos que comprenden una amplia gama de consecuencias derivadas de dichos actos las que incluyen desmotivación, depresión, impotencia y pérdida de autoestima.

\section{Bibliografía}

1. Organización Mundial de la Salud. Global Consultation on Violence and Health. Violence: a public health priority. Informe de un Grupo Científico de la OMS. Ginebra: OMS; 1996.

2. Consejo Internacional de Enfermería, CIE. Directrices para hacer frente a la violencia en el lugar de trabajo. Ed. Ginebra. 1998.

3. Poblete M, Valenzuela S. Enfermeras en riesgo: Violencia laboral con enfoque de género. Index Enferm [revista en la Internet]. 2005 mayo agosto. [acceso 28 de setiembre del 2011]; 14(51): 40-44. Disponible en: http://scielo.isciii.es/scielo.php?script=sci_arttex
t\&pid=S1 132-12962005000300008\&lng=es.doi: 10. 4321 / S1132-12962005000300008.

4. Lévano C. Enfermeras reclaman trato justo del estado. Diario la primera. Ed 002946. Perú 2011

5. Polit. y Investigación Científica en Ciencias de la Salud: principios y métodos. $6^{\text {a }}$ ed. México:

Mc. Graw - Hill; 2000.

6. Rodríguez G, Gil F. Metodología de la Investigación Cualitativa. $2^{\text {a }}$ ed. Málaga: Aljibe, S.L; 1996

7. Pérez S. La investigación cualitativa. Editorial: la Muralla, Madrid - España, 2002

8. Burns, Nancy, Kerouac Susan. Investigación en enfermería. Ed: ELSEVIER- España SA. $3^{\circ}$ ed. Madrid España. 2005

9. Sgreccia. Principios de Bioética Personalista; 2009

10. Hungler BP. Investigación científica en ciencias de la salud. $6^{a}$ ed. México: Interamericana; 2000

11. Letelier P y Valenzuela S: Violencia: Fenómeno relevante de estudio en campos clínicos intrahospitalarios de Enfermería. Revista Ciencia y Enfermería, 2002.

12. Cook y Fontaine. Enfermería psiquiátrica. $2^{a}$ ed. Mexico: Mc Graw-Hill; 2001

13. Constitución Política de Salud http:// portal.jne.gob.pe/informacionlegal/Constitucin\%2 0y\%20Leyes 1 / CONSTITUCION\%20POLITICA\%20DE L\%20PERU.pdf

14. Pardo $\mathrm{k}$, Andia $\mathrm{M}$, Rodriguez $\mathrm{A}$. Remuneraciones, beneficios e incentivos laborales percibidos por trabajadores del sector salud en el Perú: análisis comparativo entre el ministerio de salud y la seguridad social, 2009. [Artículo en la Internet]. 2011 noviembre; [acceso 31 de Octubre 
del 2012]*28(2). Disponible en: http:// www.scielo.org.pe/pdf/rins/v28n2/a29v28n2.pdf

15. Villar M, Aranaz J. Violencia en el medio hospitalario por pacientes. [Artículo en la Internet]. 2005 Dic. [acceso 31 de Octubre del 2012]*10(2). Disponible en:

http:/ / www.scsmt.cat/Upload/TextComplet/9/9 8.pdf

16. Barrera L, Villarroel G. Detección de agresión laboral al personal medico y de Enfermería del policlínico central. [Artículo en la Internet]. 2007 Marzo. [acceso 31 de Octubre del 2012]* 4(6). Disponible en: http://www.mflapaz.com/ Revista_6/revista_6_pdf/3\%20Agresion\%20laboral .pdf

17. International center for human resources in nursing. Acoso laboral en el sector salud. [Sede web]* Ginebra suiza. 20072009 [Acceso 31 Mayo de 2012] disponible en http://www.ichrn.com/publications/factsheets/FS _bullying_final-spa.pdf

18. Burgos M., Aravico K. Violencia Hospitalaria en pacientes. Cienc. enferm. [Internet]. 2003 Jun [citado 31 mayo 2012]; 9(1): 9-14. Disponible en: http:/ / www.scielo.cl/scielo.php?script =sci_arttext \&pid=S0717-95532003000100002\&lng=es. http://dx.doi.org/10.4067/S071 7955320030001 00002 .

19. Moreno, J. "Psicología de la Personalidad". 1 ed. Madrid: Thomson, 2007

20. Prensa libre. Desmotivación laboral conlleva fuerte desgaste [Sede web]* Costa Rica, 2009 [Acceso 30 Mayo de 2012] disponible en http:/ / www.prensalibre.cr/pl/suceso/2014desmotivacion-laboral-conlleva-fuertedesgaste.html 